\title{
Innovative Educational Technology for the Use of Modern CAD Systems for the Formation of Professional Competences of Students of Radio Engineering Specialties
}

\author{
Victor B. Suchkov ${ }^{1 *}$, Andrei K. Likhoedenko ${ }^{1}$, Grigory M. Seregin ${ }^{1}$, and Yury V. Karakulin ${ }^{1}$ \\ ${ }^{1}$ Bauman Moscow State Technical University, 2nd Baumanskaya str., 5/1, 105005, Moscow, Russia
}

\begin{abstract}
The basic principles of application of innovative educational technology on the basis of modern systems of automated design for higher educational institutions for students of radio engineering specialties are considered. Methods of forming competences in students for independent use of automated design systems in simulation of complex elements of autonomous information and control systems are shown. Features of modern CAD systems such as PTC Creo Parametric and Altair FEKO are described for their opportunities in teaching process in technical university. The results of simulation and calculations obtained by students during their individual tasks are given. 3D-models of microwave devices developed by students are discussed for formation of competences in electromagnetic simulation by numerical methods in special CAD systems. Especially, aspects of relationship between capabilities of students to create 3Dmodels and to calculate current distribution in microwave systems are discussed.
\end{abstract}

\section{Introduction}

The training of specialists for modern industrial clusters in Russia, capable of solving complex modern problems of designing, constructing, researching and developing complex technical systems, should be organized taking into account global trends in the development of both technology and higher professional education [1-3]. Today, one of the urgent problems is the problem of providing enterprises and organizations with staffing capable of ensuring sustainable dynamics in the development of the industrial cluster in the face of fierce competition with foreign companies. The personnel problem is becoming global across the country, for the solution of which purposeful and concrete actions are required both in the field of industrial policy and in the field of higher education.

The system of higher professional education, meeting the needs of modern industrial development, often follows the path of narrow specialization of graduated engineers, which does not allow to fully solve the problem of training highly qualified specialists [4-5]. Modern conditions are characterized by the specialization of knowledge, when, along with

* Corresponding author: vbs-2014@bmstu.ru 
deep highly specialized knowledge, future specialists receive broad methodological training on the basis of specialized educational programs. These programs are based on the interdisciplinary principle of training, intensive academic and scientific work on individual curricula. At the same time, the educational process should be integrated with intensive research activities with close connection with the industry and taking into account its needs. Due to the integration of educational and research activities, the individualization of curricula, the high level of teaching staff, the necessary material and technical equipment in Bauman Moscow State Technical University (BMSTU) became able to train highly qualified specialists, bachelors and masters for industries, taking into account the development trends of modern technologies.

In BMSTU training for specialists in the field of designing airborne radar systems is carried out by the department "Autonomous information and control systems" (AIUS). Training of highly qualified specialists in the field of on-board control systems and radar sensors, owning modern methods of research, modeling and design, can be carried out during all stages of education according to special programs in accordance with the needs of enterprises of industrial clusters.

The complexity and innovativeness of the created technical systems determines the appearance in the curriculum of the department of disciplines related to the development and practical application of modern computer-aided design systems, and in particular:

- Computer graphics;

- Microwave devices and antennas;

- Fundamentals of computer modeling and design of electronic equipment;

- Automated design of radar-location autonomous information and control systems.

It would be interesting to consider the opportunity of use CAD systems in the learning process to form special competence for students of radio engineering specialties.

\section{Theoretical background}

The purpose of studying the disciplines "Computer Graphics" and "Fundamentals of Computer Modeling and Designing of Radioelectronic Tools" is to master the theoretical and practical foundations of using computer-aided design packages focused on application in engineering, and to study the rules and skills for the production of drawing and design documentation in electronic form. As part of the disciplines, the basics of CAD, sketching and solid-state spatial modeling of parts in the computer-aided design package PTC Creo Parametric are considered.

As part of the study of PTC Creo Parametric CAD systems, students will develop competencies in the design and construction of individual nodes and path elements of microwave devices (waveguide devices and antennas) that are part of modern autonomous information and control systems. A distinctive feature of the models of microwave nodes created in modern CAD systems is the ability to convert 3D models into a polygonal model, which is a finite collection of the same flat elements, the number and shape of which is determined by the developer.

When carrying out calculations of microwave devices, a polygonal model should be used, which represents the surface of the object in the form of a set of plane triangles. The polygonal mesh of microwave devices in modern CAD systems is always created in relation to the requirements of the finite element method. The polygonal model should maximally correspond to the real geometric image of the object. In PTC Creo Parametric [6], a virtual geometric model of the microwave device is formed according to general drawings, and a polygonal model is created on the basis of the virtual model.

The purpose of studying the disciplines "Microwave and Antenna Devices" and "Computer-Aided Design of radar-location AIUS" is to master the theoretical and practical 
fundamentals of microwave devices and antennas, to master the principles and methods of computer-aided design of radar-location AIUS. Within the framework of the disciplines, the basics of CAD of electrodynamic modeling of microwave devices and antennas in the Altair FEKO computer-aided design package are considered.

CAD Altair FEKO is a complex tool for solving a wide range of tasks in the field of analysis of antennas and microwave devices. The Altair FEKO package uses the entire spectrum of the current numerical methods of electrodynamics to solve the problems of modeling microwave devices. The methodology for calculating microwave devices in the Altair FEKO system is based on the method of moments (MoM). The practical application of the method of moments to the solution of the problem of calculating a microwave device (antenna) defined as a facet geometric model was obtained by Rao, Wilton and Glisson [7]. The set of basis functions RWG proposed by the authors is a generally accepted world standard for solving electromagnetic problems of a wide class by the method of moments, including the problem of antenna radiation.

The outer surface of the microwave device (antenna) is represented as a set of plane polygons (Figure 3). The method of moments is based on the numerical solution of the integral equation of the electric field (IEEF) as applied to a three-dimensional polygonal grid. IEEF is used to determine currents on the surface of the antenna. In accordance with the method of moments, the surface current density on the surface of the antenna is defined as a superposition of local surface current densities arising on the fractures of the surface specified by the facet model [8-9].

In accordance with the method of moments, the surface current density on the surface of the antenna is defined as a superposition of local surface current densities arising on the fractures of the surface specified by the facet model [7]:

$$
\vec{J}=\sum_{n} I_{n} \vec{f}_{n}
$$

where $I_{n}$ are unknown values f current densities on triangular elements of the facet model, $\vec{f}_{n}$ are basis functions determined from the facet representation of the surface.

As a result, the task of investigation of currents on the surface of the body is reduced to the matrix equation:

$$
[Z][I]=[V]
$$

where $Z_{m n}$ are interaction matrix elements, $V_{m}$ are excitation vector elements; [I] is a vector of current densities.

Obviously, to solve the problem of calculating currents by the method of moments, it is necessary to calculate a matrix of dimension, where $N$ is the number of facets of the model. It is necessary to calculate $N^{2}$ interactions between the facets, each of which is described by double integrals. So, if the antenna model consists of $N=10^{4}$ facets, then when finding currents on the surface, the calculation of $N^{2}=10^{8}$ interactions will be required. Taking into account the aspect that double integrals [7] are solved numerically, the time spent on calculations can range from several days to several weeks, depending on the type of computing tool.

Currently, this problem has been solved by using a modification of the method of moments - the fast multipole method (MLFMM). The essence of the method is that the interaction is sought not between the triangles of the model, but between the groups of triangles combined into projection parallelepipeds. As a result, the required number of interactions, which is calculated in the process of solving the integral equation based on the matrix equation, is reduced to $N \lg N$. Then, for an object consisting of $N=10^{4}$ facets, 
interactions are determined as $N \lg N=4 \cdot 10^{4}$, i.e. the performance of the MLFMM method is increased by several orders of magnitude compared to the method of moments. However, the lower limit of applicability of this method is the size of the interaction matrix Z, which should be at least $20 \%$ of the size of the interaction matrix when using the MoM.

\section{Results and discussion}

Creation of 3D models of a microwave device in Creo Parametric is carried out using solidstate modeling tools. At the first stage, geometric models of the main parts of the microwave device are created. At the second stage, an assembly file of a complex microwave device is formed based on the created models of individual parts. At the third stage, it is recommended to combine the assembly model of the object into one part, which will be further used to create a mesh model.

The main advantage of using the Creo Parametric package is a very high degree of reliability of the geometric model of the target to its real prototype. For an example, Figures 1-2 show 3D models of a waveguide transition with an H-sectorial horn and a conical horn antenna, developed by students in the framework of laboratory studies in the above disciplines. Figures 3-4 shows the polygonal models of the waveguide transition with an $\mathrm{H}$ sectorial horn and a conical horn, which were formed by students of the Department of AIUS.

To create a polygonal model of the object, it is necessary to establish the maximum size of the triangular mesh element, which is selected from the electrodynamic criteria for microwave simulation. The maximum size of the triangle is selected in Creo Parametric in those units in which the dimensions of the object are specified.

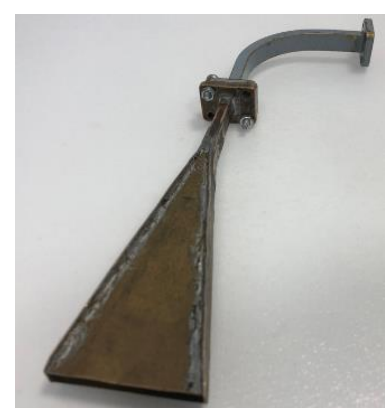

(a)

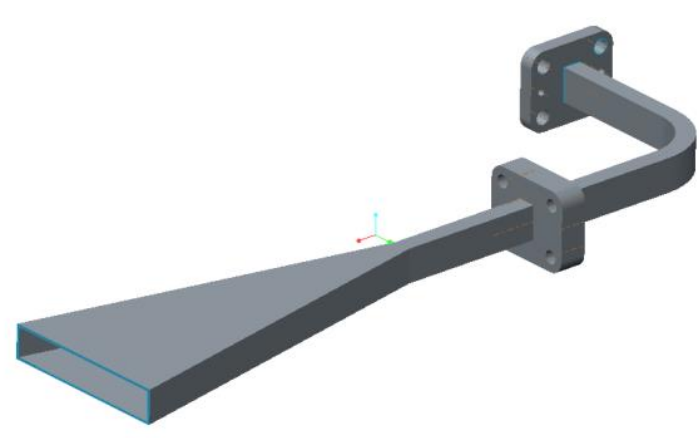

(b)

Fig. 1. Prototype (a) and 3D model (b) of a waveguide transition with an H-sectorial horn developed in PTC Creo Parametric.

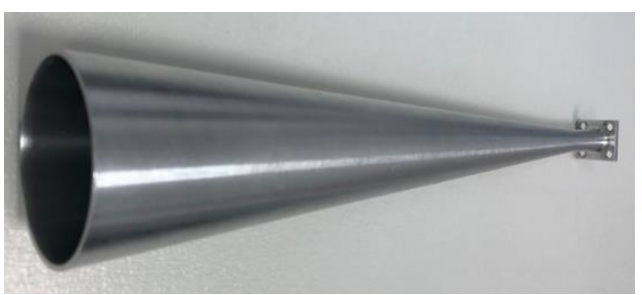

(a)

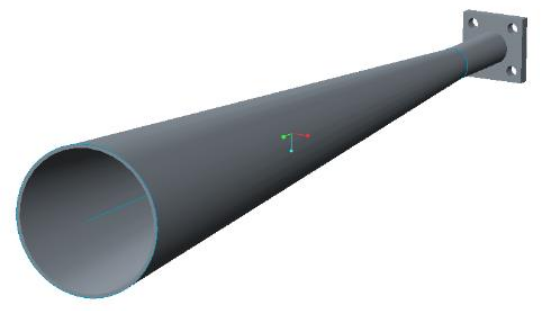

(b)

Fig. 2. Prototype (a) and 3D model (b) of a conical horn. 


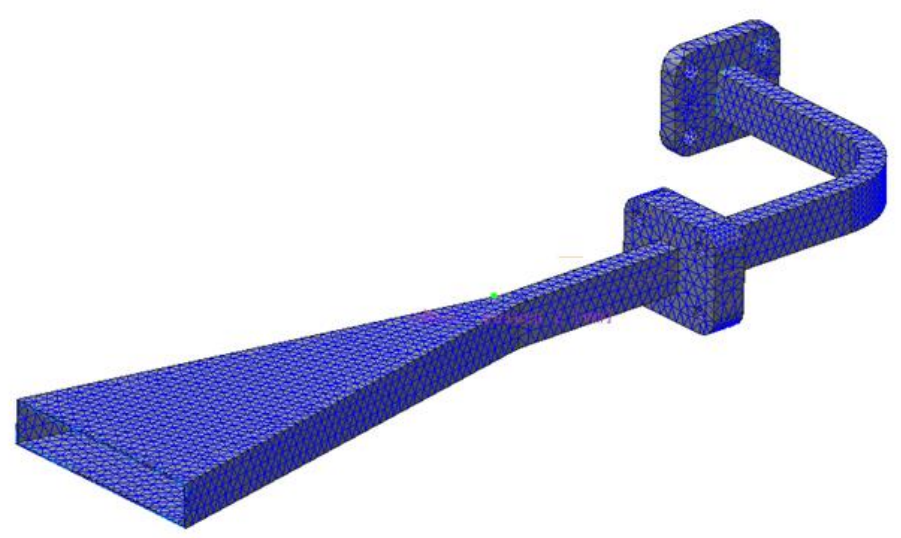

Fig. 3. Polygonal models of a waveguide transition with $\mathrm{H}$-sectorial horn.

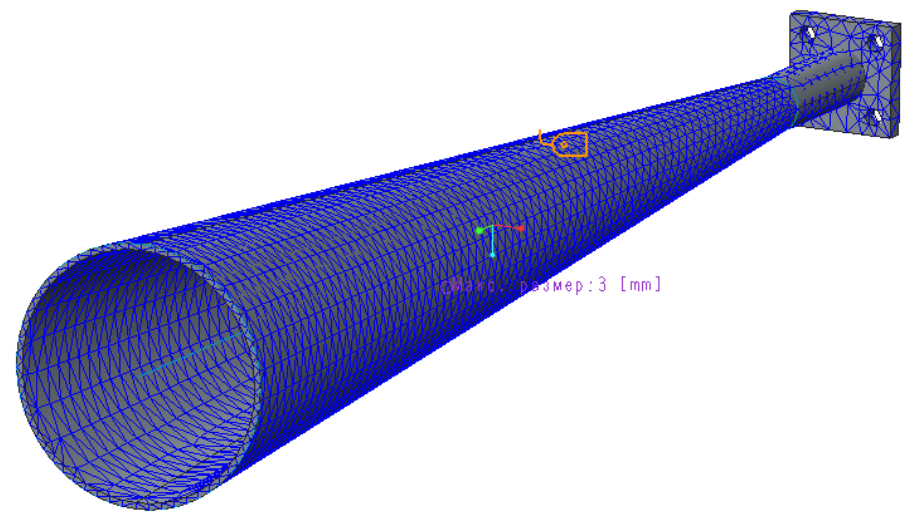

Fig. 4. Polygonal models of a conical horn.

With a decrease in the size of the triangle compared to the size of the object, the number of triangles describing its surface will increase sharply, which will increase the time it takes to create a mesh model and require significant computer computing resources. When choosing the size of a triangular mesh element, it is recommended to use local restrictions in individual surfaces of the object, which should be described most accurately. When creating a solid-state mesh, PTC Creo Parametric uses the Boundary Mesh boundary mesh.

Figures 5-6 shows the current distributions (1-2) for polygonal models of a circular waveguide at a frequency of $100 \mathrm{MHz}$ and in a power splitter at a frequency of $9 \mathrm{GHz}$, which were formed by students of the AIUS department during laboratory work in the disciplines "Microwave and Antenna Devices" and "Automated design of location AIUS ".

Thus, the following requirements can be formulated to students for choosing a method for calculating of the microwave devices and antennas [10-14] in the Altair FEKO:

- for devices whose facet model is made up of less than 30,000 triangles, it is rational to use the MoM moment method, which allows the most adequate and accurate calculation of the antenna patterns of a complex spatial and electrophysical structure in the working frequency band;

- for antennas whose polygonal model is made up of more than 30,000 triangles, from the point of view of optimizing computer computing resources, it is advisable to use 
multilevel fast multipole method (MLFMM), which allows more efficient than the moment method to calculate the microwave device or antenna pattern of complex structure.

In developing new antennas especially for pulsed transceiver modules, it is a great importance to achieve the best coordination of the antenna itself and the input path of the transceiver module. Therefore, when designing the antenna, it seems appropriate to implement the improved method of parametric synthesis based on the following principles:

a) at the first step, primary antenna modeling is carried out when the basic antenna design is formed, which in principle can provide the parameters specified for device (operating frequency band, radiation pattern width, level of SWR) in the required overall dimensions;

b) at the second step, the antenna matching circuit is modeled, the purpose of which is to optimize the antenna design parameters taking into account the achievement of the best matching characteristics (the minimum possible value of the reflection coefficient at the antenna input);

c) at the third step, modeling the characteristics of the antenna with the optimal design is carried out taking into account the matching circuit, including the construction of the antenna pattern.

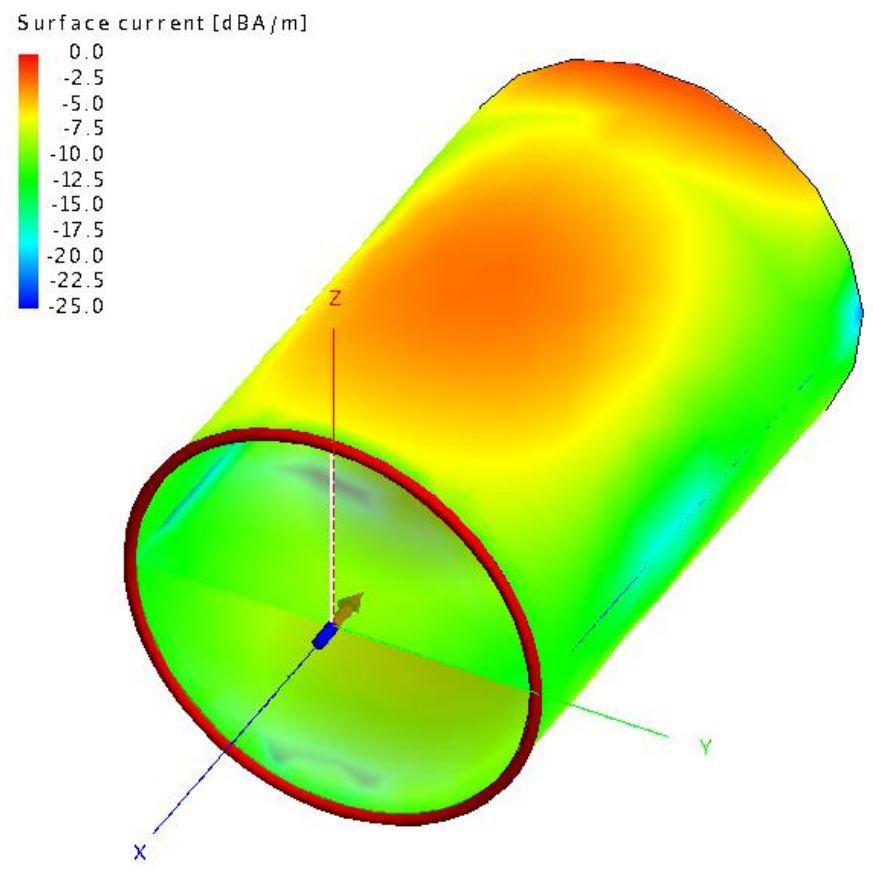

Fig. 5. Distribution of current amplitudes in a circular waveguide at a frequency of $100 \mathrm{MHz}$.

At the step of initial modeling of antennas, it seems appropriate to use the capabilities of the modern Antenna Magus, which is the most advanced antenna design tool and offers a choice of the topology model of the required emitter from the existing database. In this case, the synthesis of the emitter topology is carried out according to a formalized technical task, and the means for constructing the UWB antenna topology take into account the type of excitation.

As a result of modeling in Antenna Magus, the topology of the basic design of the Vivaldi antenna was obtained (Fig. 7), which has the following characteristics:

- dimensions of the antenna 260 to $450 \mathrm{~mm}$;

- material FR4;

- dielectric thickness $1.5 \mathrm{~mm}$; 
- type of connector is SMA.

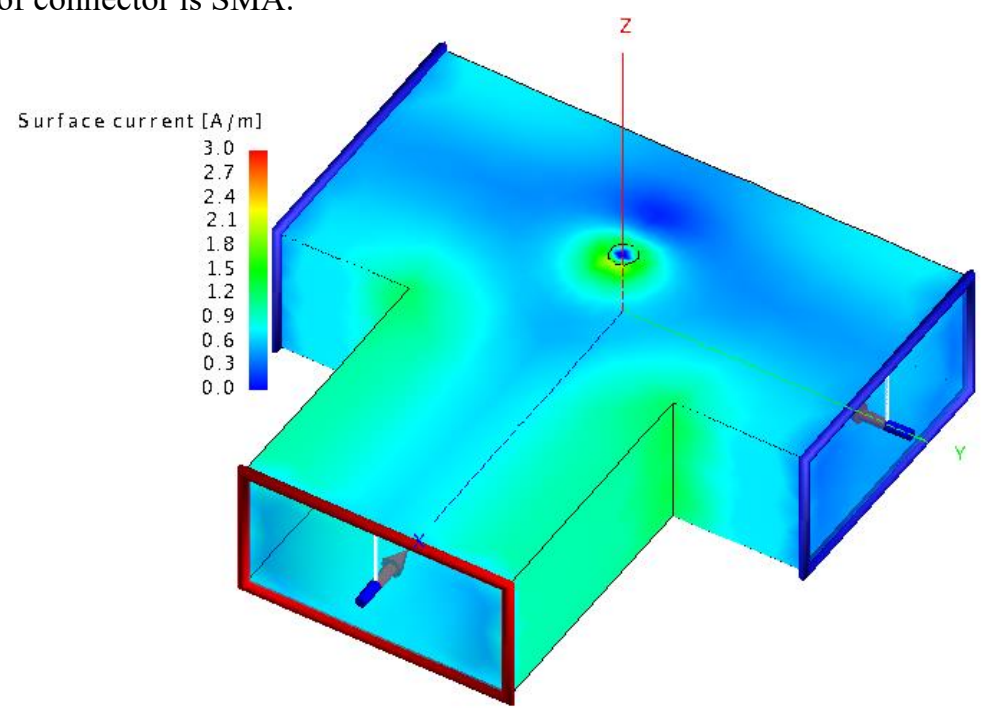

Fig.6. Distribution of current amplitudes for power divider at a frequency of $9 \mathrm{GHz}$.

In Fig. Figure 7 shows the implementation of the Vivaldi antenna bottom plate of the basic design obtained during the initial modeling phase in Antenna Magus at frequency 3 $\mathrm{GHz}$.

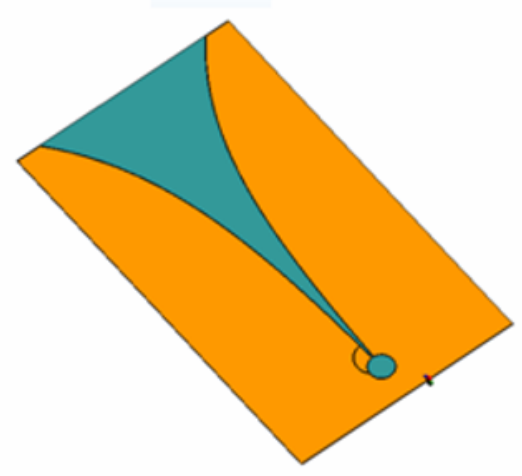

(a)



(b)

Fig. 7. Vivaldi antenna implementation (a) and its pattern at frequency of $3 \mathrm{GHz}(b)$.

Vivaldi antenna matching circuit simulation at the second stage of modeling is carried out in the Optenni Lab, which allows matching high-frequency antennas. Obviously, when tuning antennas Vivaldi antennas must be applied matching circuit. At the same time, the Optenni Lab offers a simple and convenient tool for this.

The antenna designer sets the working frequency band and the number of matching elements. As a result of setting the initial data for modeling, the Optenni Lab searches for options for optimized combinations of matching circuits to fulfill the given requirements. After that, the developer must choose the most suitable version of the matching circuit. The result of modeling the Vivaldi antenna reflection coefficient by input for the basic antenna 
design and after optimization in Optenni Lab, taking into account the matching circuit, is shown in Fig. 8.

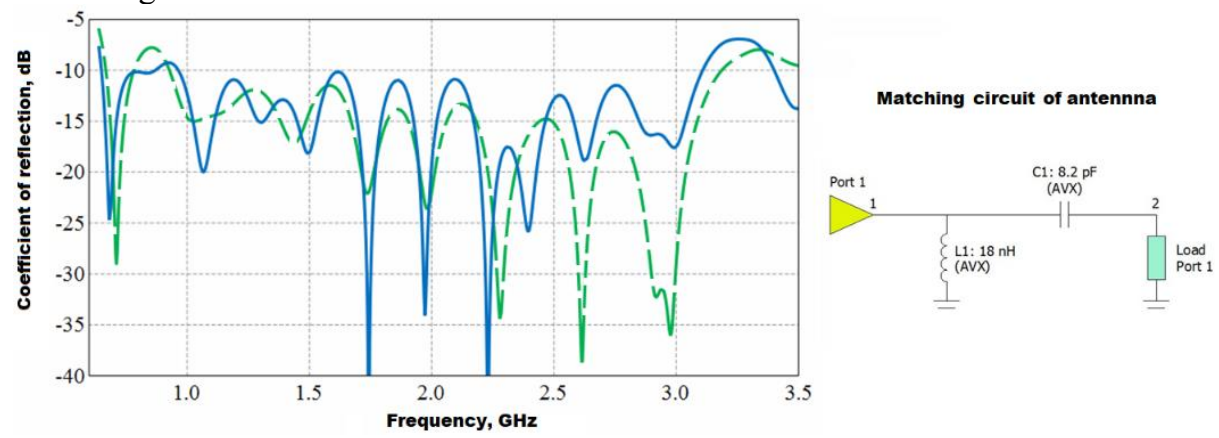

Fig. 8. The dependence of the reflection coefficient at the input of the Vivaldi antenna on frequencies (solid line - basic design from Antenna Magus, dashed line - optimized matching circuit at Optenni Lab).

As can be seen from the graphs in Fig. 8 after optimization, taking into account the matching circuit, the value of the reflection coefficient at the antenna input decreased on average by $2-5 \mathrm{~dB}$ compared to the basic design.

Thus, parametric studies of the characteristics of the Vivaldi antenna for various types of structures were carried out, it was determined that this type of antennas is implemented with small weight and size characteristics. Technique has been developed to create the optimal Vivaldi antenna design by the minimum value of the standing wave coefficient in the working frequency band and numerically simulate its radiation pattern. It is shown that when developing Vivaldi antennas, it is necessary to separately model the matching chains in order to reduce the value of the standing wave coefficient in the working frequency range.

So opportunities of PTC Creo Parametric and Altair FEKO for the formation of competencies among students of radio engineering specialties in self-modeling, designing and development of design documentation of the constituent elements of microwave devices and antenna systems AIUS are shown. The acquired competencies can be used by future specialists in the development of the components of modern AIUS operating in the microwave range.

\section{Conclusion}

The suggested innovative educational technology on the basis of modern systems of automated design allows to acquire skills of rationally using software when solving theoretical and practical problems. The use of modern CAD systems is acceptable for the formation of professional competence of students of radio engineering specialties in the framework of the formation of innovative educational technology. Formation of competencies among students of radio engineering specialties in self-modeling, designing and development of design documentation for the constituent elements of microwave devices and antenna systems is based on PTC Creo Parametric. The use of the acquired competencies for working in CAD 3D-modeling is necessary at the next stages of education for the calculation of electrodynamic models of microwave devices. The formation of competencies among students of radio engineering specialties with use of Altair FEKO is based on development of the independent choice of the method of numerical modeling of antennas and microwave devices, optimization of the design and characteristics of microwave devices. 


\section{References}

1. A.A. Aleksandrov, K. Fang, A.V. Proletarsky, K.A. Neusypin, Conception of complex continuous education with innovative information technologies, Proceedings of 2 nd International Conference on Education and Education Management, p. 374 (2012)

2. D.H. Chen, G.L. Yang, A.V. Proletarsky, K.A. Neusypin, Implementation of the Innovative Concept of Sustainable Education in the Engineering Center of Avionica, Proceedings of 4th International Conference on Advanced Education and Management, p. 361 (22017)

3. V.N. Zimin, T.Yu. Tsibizova, E.V. Chernega, D.A. Sergeev, O.M. Avgustan, Podgotovka inzhenernykh kadrov dlya tsifrovoj ehkonomiki Rossii [Preparation of engineering personnel for the digital economy of Russia], Moscow, p. 176 (2017)

4. A.A. Aleksandrov, K.A. Neusypin, B.V. Padalkin, L.G. Popovich, A.V. Proletarsky, Voprosy teorii i realizatsii nepreryvnogo obrazovaniya [Theory and Implementation of Continuing Education]. Moscow, p. 272 (2012)

5. A.A. Aleksandrov, A.V. Proletarsky, K.A. Neusypin, Kontseptsiya vzaimodejstviya MGTU im. N.E. Baumana s predpriyatiyami raketno-kosmicheskoj otrasli $v$ voprosakh tselevoj podgotovki inzhenerov $i$ nauchnykh kadrov [The concept of interaction BMSTU with enterprises of the rocket and space industry in matters of targeted training of engineers and scientists], European Social Science Journal. 1, p. 121 (2013)

6. A.B. Borzov, V.B. Suchkov, B.I. Shakhtarin, Yu.A. Sidorkina, Mathematical Modeling and Simulation of the Input Signals of Short Range Radar Systems, Journal of Communications Technology and Electronics, 59(12), p. 1356 (2014)

7. S.M. Rao, D.R. Wilton, A.W. Glisson, Electromagnetic scattering by surfaces of arbitrary shape IEEE Trans, Antennas Propagat, 30, p. 409 (1982)

8. D. Gibbson, The Method of Moments in Electromagnetics [Chapman\&Hall CRC], p. 594 (2008)

9. V.B. Suchkov, A.B. Borzov, K.P. Likhoedenko, G.M. Seregin, Applications of shortrange radars on the base of ultrashort pulse single chip, Proceedings of the 3rd IEEE International Conference on Control Science and Systems Engineering, p. 461 (2017)

10. Dennis M. Sullivan, Electromagnetic Simulation Using the FDTD Method, NY:IEEE Press, p. 165 (2000)

11. Allen Taflove, Susan Hagness, Computational Electrodynamics: The Finite-Difference Time-Domain Method, NY:Artech House, p. 467 (2000)

12. Q. Xianming, Z.N. Chen, M. Chia, Characterization of ultrawideband antennas using transfer functions, Radio Science, Vol. 41, №.1, pp. 1-10 (2006)

13. W. Wiesbeck, G. Adamiuk, C. Sturm, Basic Properties and Design Principles of UWB Antennas, Proceedings of the IEEE, Vol. 97, № 2, pp. 372-385 (2009)

14. Cam Nguyen, Jeongwoo Han, Time-Domain Ultra-Wideband Radar, Sensor and Components, New York: Springer, p. 133 (2014) 